\title{
Feedback control of flow vorticity at low Reynolds numbers
}

\author{
Maria Zeitz ${ }^{1, a}$, Pavel Gurevich ${ }^{2, b}$, and Holger Stark ${ }^{1, c}$ \\ 1 Institut für Theoretische Physik, Technische Universität Berlin, Hardenbergstraße 36, 10623 Berlin, Germany \\ 2 Institut für Mathematik 1, Freie Universität Berlin, Arnimallee 2-6, 14195 Berlin, Germany
}

Received 5 December 2014 and Received in final form 27 February 2015

Published online: 30 March 2015

(C) The Author(s) 2015. This article is published with open access at Springerlink.com

\begin{abstract}
Our aim is to explore strategies of feedback control to design and stabilize novel dynamic flow patterns in model systems of complex fluids. To introduce the control strategies, we investigate the simple Newtonian fluid at low Reynolds number in a circular geometry. Then, the fluid vorticity satisfies a diffusion equation. We determine the mean vorticity in the sensing area and use two control strategies to feed it back into the system by controlling the angular velocity of the circular boundary. Hysteretic feedback control generates self-regulated stable oscillations in time, the frequency of which can be adjusted over several orders of magnitude by tuning the relevant feedback parameters. Time-delayed feedback control initiates unstable vorticity modes for sufficiently large feedback strength. For increasing delay time, we first observe oscillations with beats and then regular trains of narrow pulses. Close to the transition line between the resting fluid and the unstable modes, these patterns are relatively stable over long times.
\end{abstract}

\section{Introduction}

Microfluidic devices offer the possibility to shape and engineer the flow of Newtonian and complex fluids on the micron scale at low Reynolds number Re [1-3] but also in the inertial regime [4]. For example, for mixing simple Newtonian fluids at low Re, turbulence is not available and, instead, one uses sophisticated channel designs and time-dependent electric or magnetic fields to create persistent mixing patterns $[1,5]$. Such patterns also occur in viscoelastic polymeric fluids [6] due to elastic instabilities $[7,8]$ which ultimately lead to turbulent flow patterns $[9,10]$. In this article, we present new concepts for designing fluid flow at low Reynolds numbers using methods from feedback control which are not common to the microfluidics community. Our aim is to initiate novel experiments in this direction. Instead in microfluidic devices the experiments can also be performed in macroscopic systems with sufficiently large shear viscosity such that the Reynolds number is low.

We illustrate our control methods for the most simple fluid, the Newtonian fluid, and choose a two-dimensional circular geometry that can be realized by a long rotating cylinder similar to the Taylor-Couette flow [11]. The fluid vorticity obeys a diffusion equation and we can focus on introducing and exploring the features of our feedback

\footnotetext{
a e-mail: maria.zeitz@tu-berlin.de

b e-mail: gurevich@math.fu-berlin.de

c e-mail: holger.stark@tu-berlin.de
}

control strategies. They can then be applied to more complex or viscoelastic fluids such as polymeric liquids [12].

We sense the mean fluid vorticity in one part of the circular geometry also called sensing area. Using either hysteretic or time-delayed feedback control, the mean vorticity determines the angular velocity of the circular boundary and thereby is fed back into the system. There is no predefined reference state, which we drive the system into using, for example, the static channel architecture of a microfluidic device or external fields. Instead, our system with feedback control is self-adaptive and generates selfregulated flow patterns.

We will present and discuss two feedback strategies. First, we will introduce hysteretic feedback control where the mean vorticity initiates discrete switching events for the angular velocity of the circular boundary. This is very similar to temperature control by a thermostat which switches heating on and off [13-17]. We will demonstrate that self-regulated oscillations occur, the frequency of which can be tuned over several orders of magnitude by choosing appropriate system parameters.

Second, we will investigate how time-delayed feedback control can be used to design fluid flow. Originally, it was invented to control chaos and to stabilize unstable periodic orbits (Pyragas control) [18]. More recently, time-delayed feedback has been demonstrated to stabilize unstable fixed points in a dynamical system [19]. The control scheme is generic and applicable to a large variety of experimental systems including an electronic chaos oscillator and coupled neurons [20]. It was used in a Newtonian fluid at high 


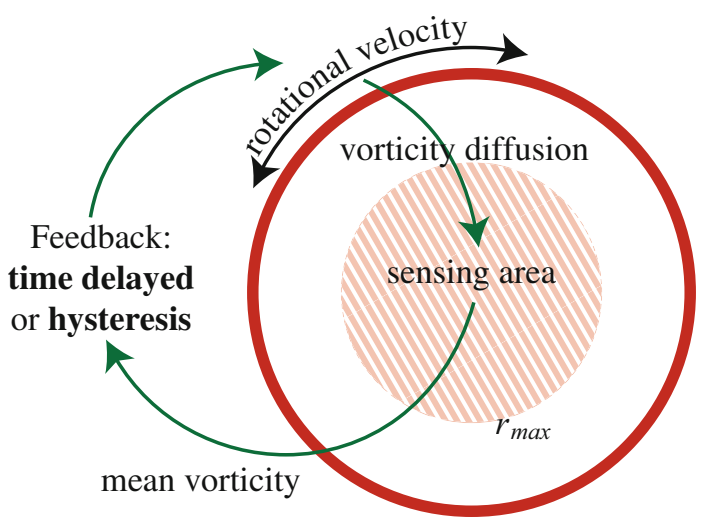

Fig. 1. Feedback scheme for vorticity diffusion in a circular domain. The mean vorticity in the sensing area, the output signal, is transformed into an input signal by feedback control with either hysteresis or time delay, which determines the angular velocity of the circular boundary.

Reynolds numbers to control the chaotic Taylor-Couette flow [21] and most recently in colloidal systems [22-24] and in liquid crystals [25]. Most often, in extended systems a local feedback scheme is used, which acts on each variable. Feedback via a global control variable has been explored in reaction-diffusion systems [26] and for Turing patterns on networks [27]. In our case the global control variable is the angular velocity of the circular boundary, which is easy to implement in an experiment. Furthermore, it is separated from the sensing area, which is still an unexplored strategy in the field of feedback control.

In the following, we identify regions in the parameter space, where time-delayed feedback destabilizes the resting fluid. Close to the transition between the stable and unstable regime long-lived oscillatory states in vorticity occur, which for increasing delay time $\tau$ exhibit beats. For $\tau$ larger than the intrinsic vortex diffusion time, they develop into bursts of narrow pulses equally spaced in time.

The article is organized as follows. In sect. 2 we describe the model system at low Reynolds numbers with all relevant equations. In sect. 3 we investigate the hysteretic feedback scheme and present both analytic and numerical results for the self-regulated oscillatory states. Then, we address time-delayed feedback in sect. 4. We first identify the fastest growing, instable mode to set up the state diagram in the parameter space. Then, we present full numerical solutions in order to identify the characteristic vorticity patterns with beats and localized pulses. We finish with a discussion.

\section{The model system}

In the following we will study a Newtonian fluid in a cylindrical geometry as shown in fig. 1. For systems on the micron scale or with sufficiently large fluid viscosity, we assume small Reynolds number and describe fluid flow by the time-dependent Stokes equations

$$
\rho \frac{\partial}{\partial t} \mathbf{v}=-\nabla p+\eta \nabla^{2} \mathbf{v}
$$

In long cylinders of radius $R$, fluid flow becomes effectively two-dimensional in the polar $r-\varphi$ plane. Taking the curl of eq. (1) and defining the vorticity $\Omega \mathbf{e}_{z}=\frac{1}{2} \operatorname{curl} \mathbf{v}$, where the unit vector $\mathbf{e}_{z}$ is perpendicular to the polar plane, we obtain the diffusion equation

$$
\frac{\partial \Omega(\mathbf{r}, t)}{\partial t}=\nabla^{2} \Omega(\mathbf{r}, t) .
$$

For incompressible fluids $\operatorname{div} \mathbf{v}=0$ holds and the fluid velocity field is fully determined by its vorticity. In the last equation we have already normalized lengths by cylinder radius $R$ and time by the diffusion time $\tau_{0}=\rho R^{2} / \eta$, which the fluid vorticity needs to diffuse through the whole system.

We will use the simple circular geometry to explore control strategies in systems at low Reynolds number such that fluid flow is laminar. Flow in the circular domain is generated by rotating the outer boundary with a rotational velocity determined through a feedback loop (see fig. 1). This creates a vortex at the boundary, which diffuses through the system. In the "sensing area" of size $A_{s}=\pi r_{\text {max }}^{2}$, an output signal is evaluated as the mean vorticity

$$
\bar{\Omega}(t)=1 / A_{s} \iint \Omega(\mathbf{r}, t) \mathrm{d} A .
$$

The output goes through a feedback scheme either with hysteresis or time delay. The feedback scheme generates an input signal which controls the rotational velocity at the boundary. This procedure defines the whole feedback loop.

Since vorticity is the same at each point of the boundary, we have an axisymmetric problem and vorticity in the circular domain only depends on the radial coordinate $r$ : $\Omega=\Omega(r, t)$. The Laplacian in the diffusion equation (2) then becomes

$$
\nabla_{r}^{2}=\frac{\partial^{2}}{\partial r^{2}}+\frac{1}{r} \frac{\partial}{\partial r} .
$$

To solve eq. (2), one has to supplement it by initial and boundary conditions. Furthermore, to have a smooth vorticity field in the center of the circular domain,

$$
\left.\partial_{r} \Omega(r, t)\right|_{r=0}=0
$$

must be fulfilled at all times. For example, when the boundary rotates with constant angular velocity $\Omega_{0}$, the fluid velocity points along the azimuthal direction with component $v_{\varphi}=\Omega_{0} r$ and the vorticity $\Omega(r, t)=\Omega_{0}$ is constant over the whole domain [28]. The feedback schemes mentioned before prescribe the vorticity at the circular boundary $\Omega(r=1, t) \equiv \Omega_{0}(t)$, which corresponds to a Dirichlet boundary condition. The value of $\Omega_{0}(t)$, the input signal, is determined from the output signal, the mean vorticity. For feedback control with hysteresis, the angular velocity of the boundary switches discontinuously between $\Omega_{0}= \pm 1$ (see sect. 3 ) and for time-delayed feedback it varies continuously with the mean vorticity in the sensing area (see sect. 4).

The operator in eq. (4) has the zeroth Bessel functions as eigenfunctions, namely of the first kind, $J_{0}(r)$, 


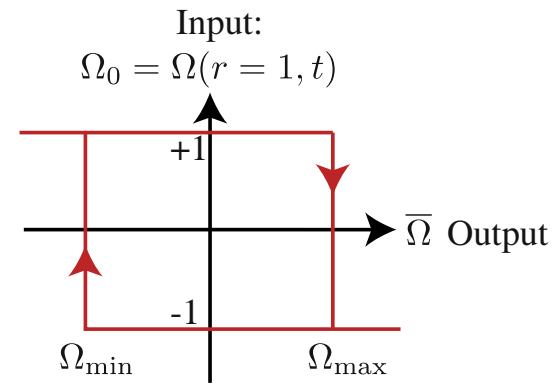

Fig. 2. Scheme for hysteretic feedback control. The output, the mean vorticity $\bar{\Omega}$, determines the input, the angular velocity of the circular boundary $\Omega_{0}=\Omega(r=1, t)$.

and the second kind, $Y_{0}(r)$, and the eigenvalue is one. Without the axial symmetry of our problem, also higherorder Bessel functions would become important. The fluid vorticity should be finite at $r=0$ and we only need to consider $J_{0}(r)$, since $Y_{0}(r)$ diverges at $r=0$. We will use this information in sect. 4.1.

\section{Feedback control with hysteresis}

In the hysteretic feedback scheme the mean vorticity $\bar{\Omega}$ basically oscillates between a minimum and a maximum value, $\Omega_{\min }$ and $\Omega_{\max }$. The input signal, the new angular velocity $\Omega_{0}$ of the boundary, depends not only on $\bar{\Omega}$ but also on the current value of $\Omega_{0}$ as fig. 2 demonstrates. If the mean vorticity falls below the lower threshold, $\bar{\Omega}(t)<\Omega_{\min }$, the angular velocity $\Omega_{0}$ is switched from -1 to +1 . The mean vorticity increases and if it becomes larger than the upper threshold, $\bar{\Omega}(t)>\Omega_{\max }$, the angular velocity $\Omega_{0}$ of the circular boundary reverses again from +1 to -1 . A whole cycle is completed, when $\bar{\Omega}(t)$ reaches $\Omega_{\text {min }}$. After some transient regime, this scheme produces a self-regulated, regular oscillation of the circular geometry if the boundary value $\Omega_{0}$ falls outside the interval $\left[\Omega_{\min }, \Omega_{\max }\right]$. Otherwise, for $\Omega_{\min }<\Omega_{0}<\Omega_{\max }$ the container rotates steadily with a constant angular velocity. In the following we present a formal analytic solution of the problem as well as concrete numerical results.

\subsection{Analytic solution}

The general solution of the diffusion equation with nonzero (non-homogeneous) boundary and intitial conditions can be formulated using Green's function $g\left(\mathbf{r}, t \mid \mathbf{r}^{\prime}, t^{\prime}\right)[29]$

$$
\begin{aligned}
\Omega(\mathbf{r}, t)= & \int_{t_{0}}^{t} \oint_{S^{\prime}}\left[g\left(\mathbf{r}, t \mid \mathbf{r}^{\prime}, t^{\prime}\right) \nabla^{\prime} \Omega\left(\mathbf{r}^{\prime}, t^{\prime}\right)\right. \\
& \left.-\Omega\left(\mathbf{r}^{\prime}, t^{\prime}\right) \nabla^{\prime} g\left(\mathbf{r}, t \mid \mathbf{r}^{\prime}, t^{\prime}\right)\right] \cdot \mathbf{n} \mathrm{d} S^{\prime} \mathrm{d} t^{\prime} \\
& +\int_{V^{\prime}} \Omega\left(\mathbf{r}^{\prime}, t_{0}\right) g\left(\mathbf{r}, t \mid \mathbf{r}^{\prime}, t_{0}\right) \mathrm{d} V^{\prime} .
\end{aligned}
$$

The boundary values for $\Omega$ and its derivatives contribute via the first integral, where $\mathbf{n}$ is the surface normal, and the initial value at time $t_{0}$ appears in the second integral. Green's function solves the diffusion equation with zero (homogeneous) boundary condition. In our case, for the axisymmetric system the boundary condition is $g(r=$ $\left.1, t \mid r^{\prime}, t^{\prime}\right)=0$ and Green's function reads [29]

$$
\begin{aligned}
g\left(r, t \mid r^{\prime}, t^{\prime}\right)= & \frac{\Theta\left(t-t^{\prime}\right)}{\pi} \sum_{n=1}^{\infty} \frac{J_{0}\left(k_{n} r^{\prime}\right) J_{0}\left(k_{n} r\right)}{J_{1}^{2}\left(k_{n}\right)} \\
& \times \exp \left[-k_{n}^{2}\left(t-t^{\prime}\right)\right] .
\end{aligned}
$$

Here $J_{m}(x)$ is the $m$-th Bessel function of the first kind and $k_{n}$ denotes the $n$-th positive root of $J_{0}\left(k_{n}\right)=0$. In our case, we only prescribe the surface value $\Omega_{0}$ at $r=1$ and eq. (5) becomes

$$
\begin{aligned}
\Omega(r, t)= & -\left.2 \pi \int_{t_{0}}^{t} \Omega_{0}\left(t^{\prime}\right) \partial_{r^{\prime}} g\left(r, t \mid r^{\prime}, t^{\prime}\right)\right|_{r^{\prime}=1} \mathrm{~d} t^{\prime} \\
& +2 \pi \int_{0}^{1} \Omega\left(r^{\prime}, t_{0}\right) g\left(r, t \mid r^{\prime}, t_{0}\right) r^{\prime} \mathrm{d} r^{\prime} .
\end{aligned}
$$

Since in the hysteretic feedback control, $\Omega_{0}$ at the boundary switches between +1 and -1 at times $t_{N}$, we construct the overall solution piecewise:

$$
\Omega(r, t)= \begin{cases}\Omega_{I}(r, t), & \text { if } 0<t<t_{I}, \\ \Omega_{I I}(r, t), & \text { if } t_{I}<t<t_{I I}, \\ \ldots, & \\ \Omega_{N}(r, t), & \text { if } t_{N-1}<t<t_{N}, \\ \ldots & \end{cases}
$$

Suppose the system is in the $N$-th cycle with solution $\Omega_{N}(r, t)$ and the mean vorticity $\bar{\Omega}_{N}(t)$ either reaches $\Omega_{\min }$ from above or $\Omega_{\max }$ from below at the switching time $t_{N}$. Then the boundary value $\Omega_{0, N}$ at $r=1$ reverses its sign to the boundary value $\Omega_{0, N+1}$, which is either +1 or -1 . Assuming that $\Omega_{0, N}=1$ for $N=1$, the full solution in the $(N+1)$-st cycle using eqs. $(6),(7)$, and $\int_{0}^{r} J_{0}\left(k_{n} r^{\prime}\right) r^{\prime} \mathrm{d} r^{\prime}=$ $r / k_{n} J_{1}\left(k_{n} r\right)$ reads

$$
\begin{aligned}
& \Omega_{N+1}(r, t)= \\
& 2 \sum_{n=1}^{\infty}\left\{\frac{(-1)^{N+1}}{k_{n}} \frac{J_{0}\left(k_{n} r\right)}{J_{1}\left(k_{n}\right)}\left(1-\exp \left[-k_{n}^{2}\left(t-t_{N}\right)\right]\right)\right\} \\
& +2 \int_{0}^{1} \mathrm{~d} r^{\prime}\left[r^{\prime} \Omega_{N}\left(r^{\prime}, t_{N}\right)\right. \\
& \left.\times \sum_{n=0}^{\infty}\left\{\frac{J_{0}\left(k_{n} r^{\prime}\right) J_{0}\left(k_{n} r\right)}{J_{1}^{2}\left(k_{n}\right)} \exp \left[-k_{n}^{2}\left(t-t_{N}\right)\right]\right\}\right]
\end{aligned}
$$

We take here the solution $\Omega_{N}\left(r, t_{N}\right)$ as initial condition to calculate the solution $\Omega_{N+1}(r, t)$, where $t_{N}$ is the $N$ th switching time determined by $\bar{\Omega}_{N}\left(t_{N}\right)=\Omega_{\min }$ or $\Omega_{\max }$. For completeness, we give the mean vorticity in 


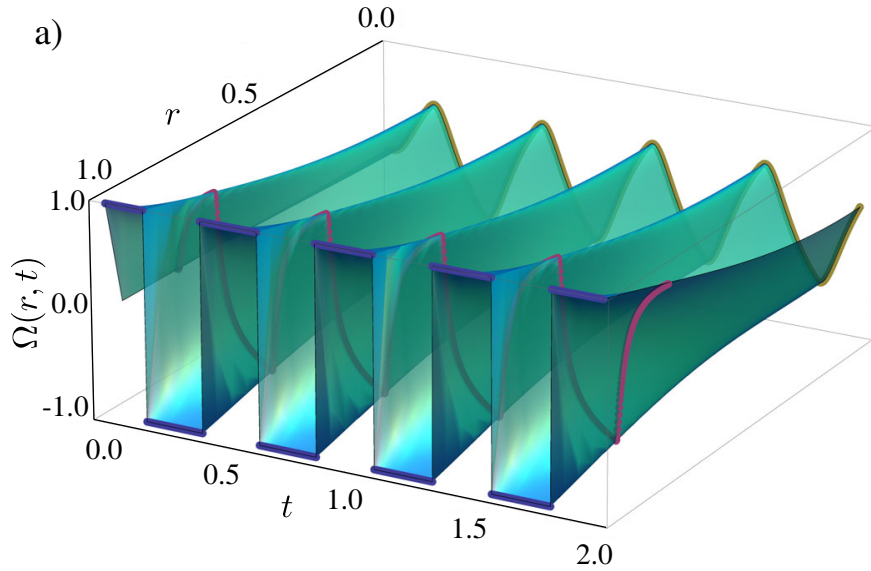

b)

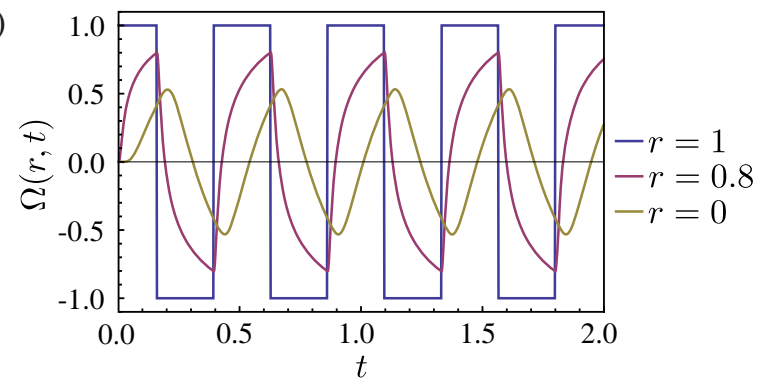

Fig. 3. a) Fluid vorticity $\Omega(r, t)$ plotted versus radial coordinate $r$ and time $t$ for $\Omega_{\max }=-\Omega_{\min }=0.5$ and $r_{\max }=0.5$. b) Fluid vorticity $\Omega(r, t)$ plotted versus $t$ for selected radial positions. The curves are also indicated in a).

the $N$-th cycle:

$$
\begin{aligned}
& \bar{\Omega}_{N}(t)=2 \int_{0}^{r_{\max }} r \Omega_{N}(r, t) \mathrm{d} r \\
& =2 \sum_{n=1}^{\infty}\left\{\frac{(-1)^{N+1}}{k_{n}^{2}} \frac{J_{1}\left(k_{n} r_{\max }\right)}{J_{1}\left(k_{n}\right)}\left(1-\exp \left[-k_{n}^{2}\left(t-t_{N}\right)\right]\right)\right\} \\
& +2 \int_{0}^{1} \mathrm{~d} r^{\prime}\left[r^{\prime} \Omega_{N}\left(r^{\prime}, t_{N}\right)\right. \\
& \left.\times \sum_{n=0}^{\infty}\left\{\frac{J_{0}\left(k_{n} r^{\prime}\right) J_{1}\left(k_{n} r_{\max }\right)}{k_{n} J_{1}^{2}\left(k_{n}\right)} \exp \left[-k_{n}^{2}\left(t-t_{N}\right)\right]\right\}\right] .
\end{aligned}
$$

This completes the analytic solution for the fluid vorticity under hysteretic feedback control. In principal, it can be implemented, for example, in Mathematica. We chose an alternative way and solved the diffusion equation numerically using a simple finite-difference method with an Euler scheme on a two-dimensional grid in polar coordinates.

\subsection{Numerical results}

In fig. 3a) we plot the numerically determined vorticity $\Omega(r, t)$ versus radial coordinate $r$ and time $t$. At the boundary at $r=1$ the discontinuous switching events of the hysteretic feedback are visible. In the bulk fluid $(r<1)$ the switching becomes smoothed out since the vorticity
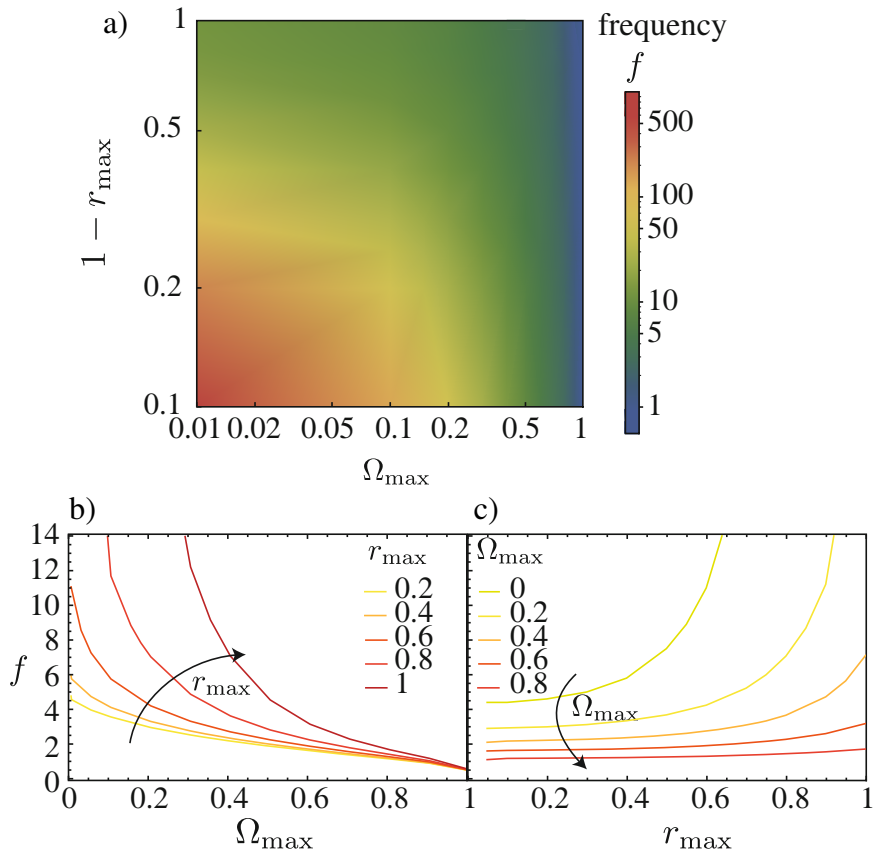

Fig. 4. a) Color-coded frequency $f$ of oscillations induced by hysteretic feedback plotted versus $\Omega_{\max }=-\Omega_{\min }$ and $r_{\max }$. All quantities are plotted on a logarithmic scale. b) Frequency $f$ versus $\Omega_{\max }$ for several $r_{\max }$. c) Frequency $f$ versus $r_{\max }$ for several $\Omega_{\max }$.

has to diffuse towards the center. The smoothing becomes better visible in fig. $3 \mathrm{~b})$, where we plot $\Omega(r, t)$ for selected radial positions. While at $r=0.8$ the sharp transitions from the hysteretic feedback at the circular boundary are still visible, the vorticity at $r=0$ shows a smooth modulation due to diffusion. The maximum of the vorticity is delayed in time relative to the sharp switching events at the boundary. The time delay is completey determined by the diffusion time $\tau_{0}$ and independent of the feedback parameters.

The hysteretic feedback leads to a self-regulated oscillation, the frequency of which is regulated by the threshold frequency $\Omega_{\max }=-\Omega_{\min }$ and the sensor size $r_{\max }$. The color-coded frequency $f$ plotted in fig. 4a) shows that the frequency variation ranges over several orders of magnitudes. Note the logarithmic scale for all quantities. Figures 4b) and c) show curves for either constant $r_{\max }$ or $\Omega_{\max }$. The general trend for frequency $f$ is clear. It increases for decreasing $\Omega_{\max }$ since the mean vorticity $\bar{\Omega}$ reaches the threshold frequency faster. Likewise $f$ also increases with increasing $r_{\max }$ since the sensing area reaches further to the boundary, where the vorticity is always larger in the non-steady state, i.e., before the system establishes the constant steady-state value $\Omega(r, t)=\Omega_{0}$ without switching events. Figure $4 \mathrm{~b}$ ) reveals that $f$ reaches finite, non-zero values for both $\Omega_{\max } \rightarrow 0$ and $\Omega_{\max } \rightarrow 1$. However, formally at $\Omega_{\max }=0$ the frequency should be infinite since $\bar{\Omega}(t)$ reaches the threshold instantaneously while for $\Omega_{\max } \geq 1$ oscillations do not occur $(f=0)$ since in this case the driving angular velocity $\Omega_{0}=1$ is always equal to or smaller than $\Omega_{\max } \geq 1$ thus the threshold is 
never reached. Likewise, for $r_{\max } \rightarrow 0$ the frequency approaches a non-zero value as illustrated in fig. 4c) while at $r_{\max }=0$ no feeback is implemented and $f$ is zero. Finally, for $r_{\max }=1$ and $\Omega_{\max } \rightarrow 0$, we expect a real divergence in $f$ since $\bar{\Omega}$ starts to increase instantaneously.

\section{Time-delayed feedback control}

We now explore our second feedback scheme to control fluid vorticity in the circular domain. We implement feedback with time delay to control the angular velocity $\Omega_{0}$ of the circular boundary. Concretely, we set $\Omega_{0}$ by comparing the mean vorticity at time $t-\tau$ with its value at time $t$ :

$$
\Omega_{0}=k[\bar{\Omega}(t-\tau)-\bar{\Omega}(t)],
$$

where $k$ is the feedback strength and $\tau$ is the delay time. This feedback scheme is typically used for stabilizing oscillating states that otherwise would be unstable $[18,19,30]$.

However, in our case we apply this scheme to a stable system and study for which parameters $k$ and $\tau$ it becomes unstable. In ref. [19] this scenario was investigated for a simple discrete system with two degrees of freedom. Classically, time-delayed feedback is implemented locally at each space point $[18,19,26]$. Here, we use a global implementation by introducing the integrated or mean vorticity to control the angular velocity $\Omega_{0}$ of the boundary, so it can easily be realized experimentally.

\subsection{Stability analysis}

To study the stability of our system against axially symmetric disturbances, we take the standard ansatz for the local vorticity

$$
\Omega(r, t)=\phi_{\alpha}(r) \mathrm{e}^{\alpha t} .
$$

Zero vorticity is stable for $\operatorname{Re}(\alpha)<0$, while for $\operatorname{Re}(\alpha)>0$ the vorticity diverges in time. In between at $\operatorname{Re}(\alpha)=0$ an oscillating state with constant amplitude occurs, which is, however, unstable against perturbations in the parameters $k, \tau$. Inserting the ansatz for $\Omega(r, t)$ in the diffusion equation (2) and using the radial part of the Laplacian (see eq. (4)), we arrive at the eigenvalue equation

$$
\left(\frac{\partial^{2}}{\partial r^{2}}+\frac{1}{r} \frac{\partial}{\partial r}\right) \phi_{\alpha}(r)=\alpha \phi_{\alpha}(r) .
$$

It is solved by the zeroth Bessel function of the first kind, $\phi(r)=J_{0}(i \sqrt{\alpha} r)$, as already discussed in sect. 2. The mean vorticity defined in eq. (3) becomes

$$
\bar{\Omega}(t)=\mathrm{e}^{\alpha t} \bar{\phi}_{\alpha},
$$

with $\bar{\phi}_{\alpha}=2 J_{1}\left(i \sqrt{\alpha} r_{\max }\right) /\left(i r_{\max } \sqrt{\alpha}\right)$. The first Bessel function of the first kind results from the integration over $r$. Using this solution in the feedback equation (11), we obtain

$$
J_{0}(i \sqrt{\alpha})=k\left(\mathrm{e}^{-\alpha \tau}-1\right) \frac{2}{i r_{\max } \sqrt{\alpha}} J_{1}\left(i \sqrt{\alpha} r_{\max }\right) .
$$

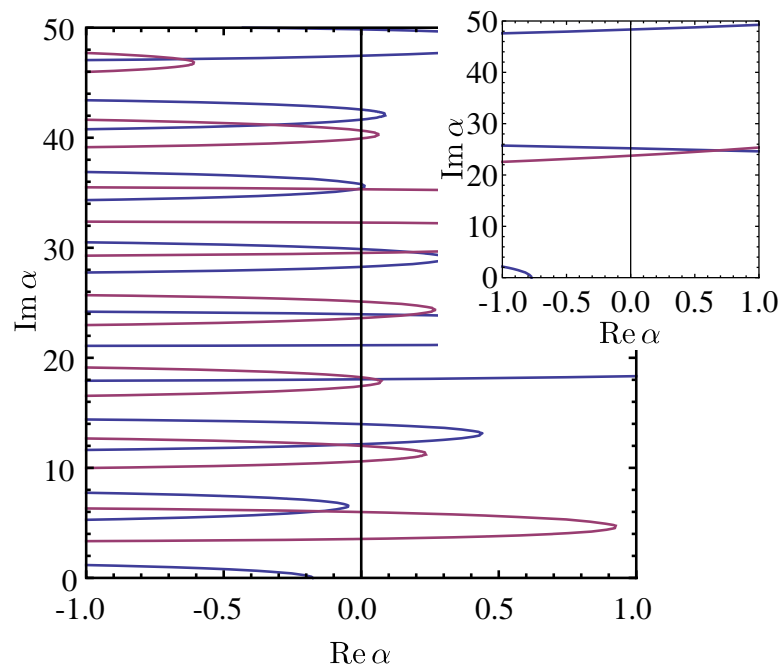

Fig. 5. Solutions of the real (blue) and imaginary (red) part of eq. (15) in the $\operatorname{Re} \alpha$ - $\operatorname{Im} \alpha$ plane. Main plot: the parameters are $k=5, \tau=1$, and $r_{\max }=0.5$. Inset: the parameters are $k=5, \tau=0.2$, and $r_{\max }=0.5$.

This implicit equation has no closed solution but it can be solved numerically in order to determine $\alpha$. Figure 5 shows curves in the $\operatorname{Re} \alpha$ - $\operatorname{Im} \alpha$ plane, which solve, respectively, the real and the imaginary part of eq. (15) for two sets of parameters. The intersection of the two curves then gives a complex $\alpha$ as a solution of eq. (15). The inset illustrates the only solution in the chosen ranges for $\operatorname{Re} \alpha$ and $\operatorname{Im} \alpha$ at a small $\tau=0.2$. Increasing $\tau$ generates more and more solutions in the same range as the main plot of fig. 5 shows. This behavior comes from the factor $\exp (-\alpha \tau)$ in eq. (15) but obviously we cannot simply rescale $\alpha$ with $\tau$. We are mainly interested in the solution with the largest real part of $\alpha$, which determines the vorticity field at long time scales, but will also comment on the influence of the other complex relaxation rates $\alpha$.

Figure 6a) shows the stability or state diagram in the $k$ - $\tau$ plane. For several $r_{\max }$ we determined the transition lines between stable and unstable states by setting $\operatorname{Re} \alpha=0$ for the largest real part of $\alpha$. To the left of the transition line $\operatorname{Re} \alpha<0$ and the resting state with $\Omega(r, t)=0$ is stable. Any disturbance with $\Omega(r, t=0) \neq 0$ decays to zero as the vorticity in fig. 6b) shows. To the right of the transition line $\operatorname{Re} \alpha>0$. The vorticity grows in time and diverges eventually as illustrated by the mean vorticity in fig. 6c). Precisely on the transition line oscillations of the vorticity with constant amplitude occur. Figure 6d) shows these oscillations at different radial coordinates. The non-zero phase shift between the curves results from the vorticity diffusion. However, the oscillations either decay to zero or diverge for any small variation in $k$ and $\tau$. Note that all curves in figs. 6b)-d) were determined by numerically solving the vortex-diffusion equation together with boundary condition (11).

The angular frequency of damped or diverging oscillations is determined by the imaginary part $\operatorname{Im} \alpha_{m}$, where $\alpha_{m}$ is again the eigenvalue with the largest real part $\operatorname{Re} \alpha_{m}$. Figure 7 shows that the range of $\operatorname{Im} \alpha_{m}$ covers 
a)
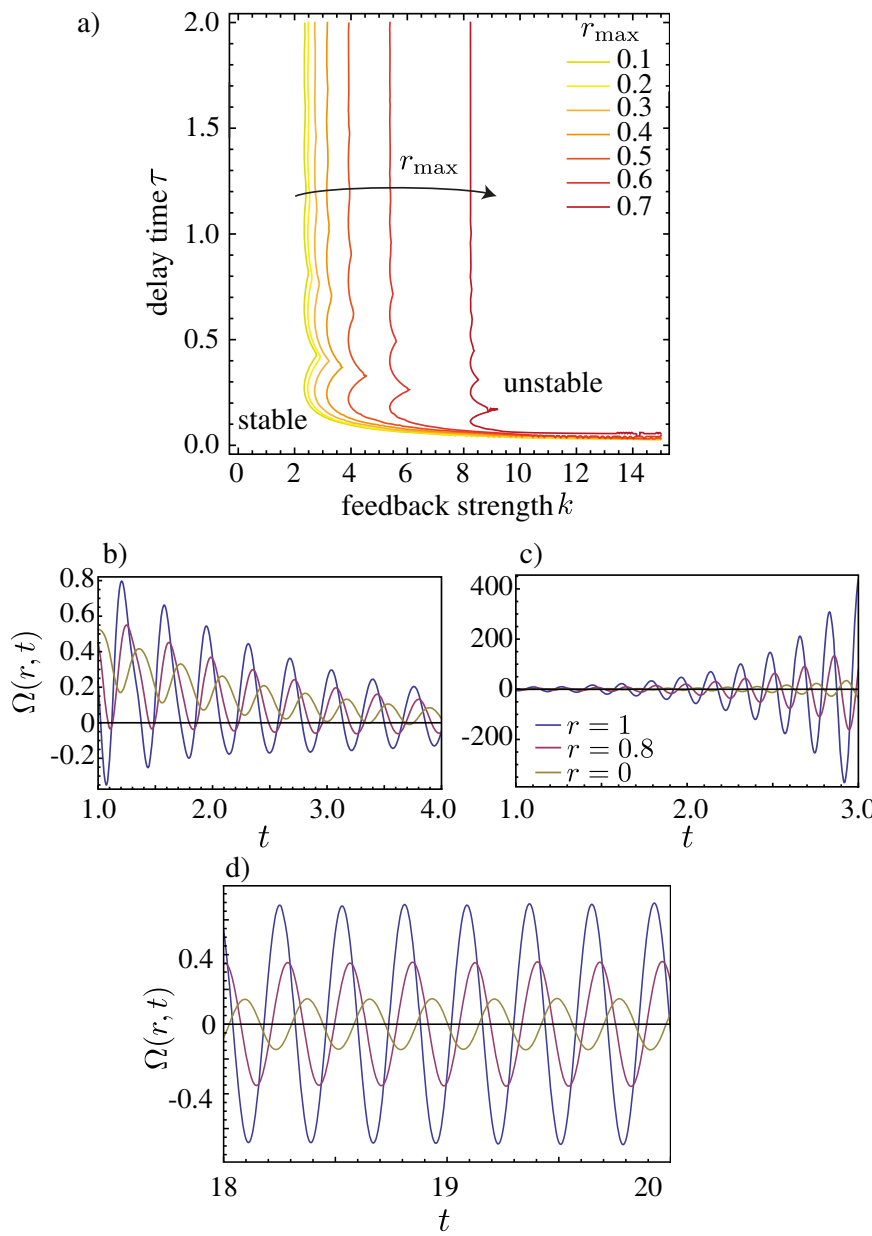

Fig. 6. a) State diagram in the $k-\tau$ plane. The transition line between stable and unstable states is indicated for different values of $r_{\max }$. b)-d) Vorticity field $\Omega(r, t)$ plotted versus time for several radial positions. b) For the stable state at $k=3$, $\tau=0.3$, and $\left.r_{\max }=0.5, \mathrm{c}\right)$ for the unstable state at $k=8$, $\tau=0.3$, and $r_{\max }=0.5$ and $\mathrm{d}$ ) on the transition line at $k=$ $3.68, \tau=0.5$, and $r_{\max }=0.5$.

nearly two orders of magnitude in the given parameter space. An overall increase with $k$ is observed. The discontinuities result when the complex solution $\alpha_{m}$ of eq. (15) jumps from one solution branch (see fig. 5) to another. When comparing with the state diagram in fig. 6 , we observe that in the stable regime disturbances are purely damped at low $k$. Increasing $k$ towards the transition line, we observe that damped oscillations start to appear, while in the unstable regime oscillations always occur.

\subsection{Beyond stability analysis}

We also solved the vortex diffusion equation numerically together with the feedback boundary condition using a simple Euler scheme. This allows to shortly discuss full solutions beyond the stability analysis that should be observable in experiments. We already discussed in connection with fig. 5 that an increase in $\tau$ increases the number

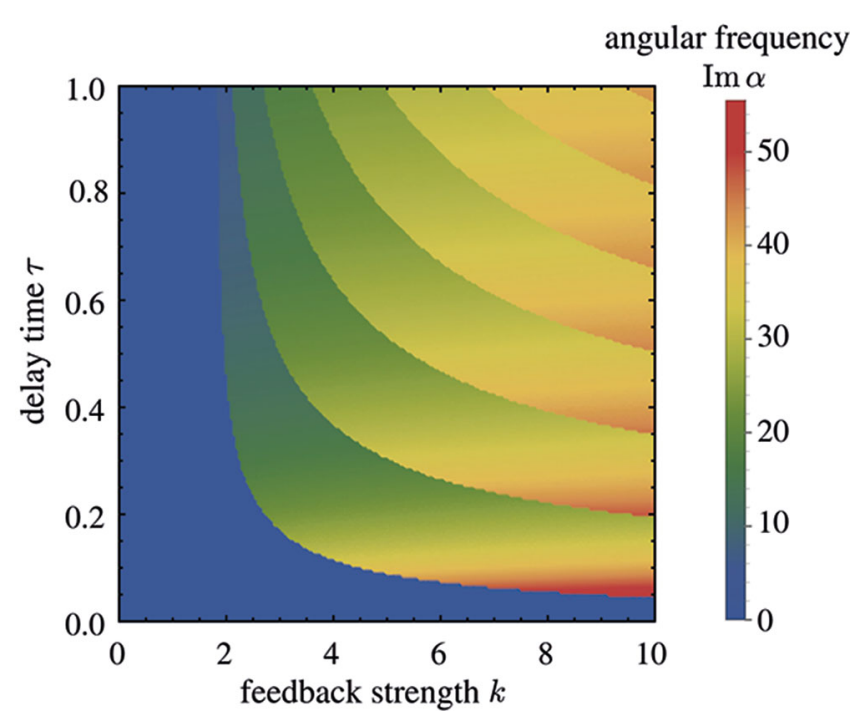

Fig. 7. Color-coded imaginary part $\operatorname{Im} \alpha_{m}$ of the exponent $\alpha_{m}$ with the largest $\operatorname{Re} \alpha_{m}$ plotted in the $k-\tau$ plane for $r_{\max }=0.5$. $\operatorname{Im} \alpha_{m}$ dominates the angular frequency of oscillations.
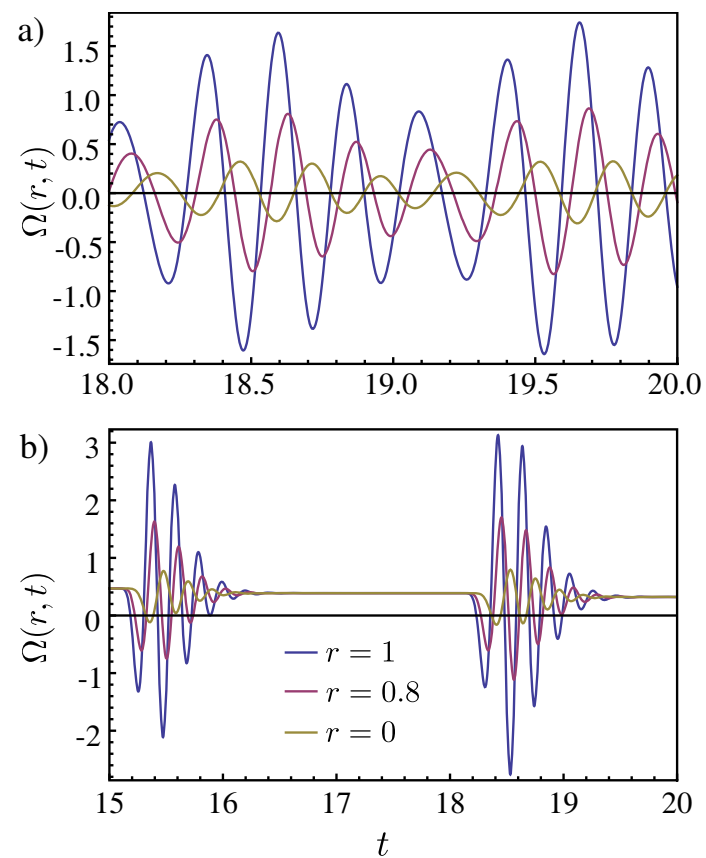

Fig. 8. Vorticity $\Omega(r, t)$ plotted versus time at several radial positions. The full numerical solution shows oscillations with beats in the unstable regime of the state diagram. Parameters are: a) $k=4, \tau=1$, and $r_{\max }=0.5$, and b) $k=4.8, \tau=3$, and $r_{\max }=0.5$.

of modes in a given range of $\operatorname{Im} \alpha$. As a result, the superposition of several of these modes creates interesting oscillation patterns with beats in the amplitude. Figure 8a) shows such beats in the vorticity at different radial coordinates close to the transition line between the stable and unstable regime at $\tau=1$. This means the overall amplitude only increases weakly. To observe such beats, the real parts of the complex rates $\alpha$ have to be similar, otherwise one mode would dominate right from the beginning. In 


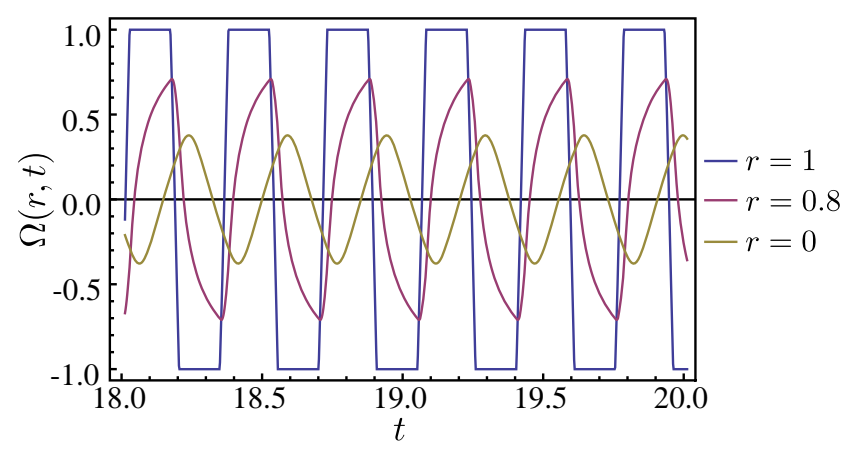

Fig. 9. Vorticity field $\Omega(r, t)$ plotted versus time for several radial positions using a cutoff $\Omega_{0, \max }=1$ in the feedback protocol as explained in eq. (16). Other parameters are $k=8$, $\tau=0.3$, and $r_{\max }=0.5$, compare to fig. $6 \mathrm{c}$ ) without the cutoff.

fig. 8b) we increase $\tau$ to 3 . Now, the superposition of several modes with similar frequencies creates narrow pulses which form a regular puls train. Since the delay time $\tau=3$ is larger than the characteristic vortex diffusion time, the system always develops a constant vorticity throughout the circular domain and between the pulses. Then, after the delay time $\tau$ the last pulse initiates a new pulse and so on. At larger $\tau$ and even further away from the transition line the real parts of the complex $\alpha$ are still small and the amplitude of the pulses only increase slowly.

Finally, a straightforward modification in the feedback protocol helps to stabilize the unstable oscillations. We simply introduce an upper bound for the magnitude of $\Omega_{0}$. Equation (11) then changes to

$$
\Omega_{0}= \begin{cases}k[\bar{\Omega}(t-\tau)-\bar{\Omega}(t)], & \text { if }\left|\Omega_{0}\right|<\Omega_{0, \max } \\ \pm \Omega_{0, \max }, & \text { else, }\end{cases}
$$

where the parameter $\Omega_{0, \max }$ sets the amplitude of the oscillations at the boundary. This is illustrated in fig. 9 .

\section{Summary and conclusions}

The aim of this artice was to introduce strategies of feedback control to design and engineer the flow of complex fluids in the regime of low Reynolds numbers. In experiments they are, for example, realizable in microfluidic geometries. To introduce and study basic features of our control strategies, namely hysteretic and time-delayed feedback, we investigated simple vortex diffusion in a circular domain. The mean vorticity in the sensing area is fed back into the system via the angular velocity of the circular boundary.

With hysteretic feedback discrete switching events of the circular boundary are initiated that generate self-regulated oscillations, the frequency of which can be tuned over several orders of magnitude via the feedback parameters. The parameters to vary are the maximum and minimum mean vorticity and the radius of the sensing area. In more complex fluids with intrinsic characteristic frequencies, we expect that the self-regulated oscillations couple to the intrinsic modes and help to explore their dynamics.

Time-delayed feedback generates unstable vorticity modes for sufficiently large feedback strength. Close to the transition line between stable and unstable states, longlived oscillatory patterns occur. For increasing delay time $\tau$ more and more oscillatory modes with complex frequencies lying close together contribute to these oscillatory patterns. At $\tau \approx 1$ they exhibit beats and for $\tau>1$ they form regular trains of narrow pulses separated by a quasistationary state of uniform vorticity. Introducing an upper bound for the angular velocity of the circular boundary, stabilizes the unstable oscillations.

Using the characteristic diffusion time $\tau_{0}=\rho R^{2} / \eta$ and the velocity of the outer boundary, $v=2 \pi f R$, where we have introduced the oscillation frequency $f$, we can rewrite the Reynolds number $\operatorname{Re}=\varrho v a / \eta$ as $\operatorname{Re}=\tau_{0} f$. In both control strategies the frequencies of the observed oscillations are around $1 / \tau_{0}$ or well above. So the interesting features, observed in this article, happen in a regime of moderate Reynolds number, where the non-linear term in the Navier-Stokes equations cannot be neglected and our analysis strictly does not apply. It will be interesting to explore in an experiment how much these features are influenced by inertial effects and monitor the new flow patterns. They should also have radial velocity components in addition to the azimuthal component treated in this article.

Complex fluids such as polymeric liquids, which exhibit elastic instabilities $[9,31]$, or phase-separating fluid mixtures [32-34] show are more complex intrinsic dynamics than the simple vortex diffusion. Certainly, the oscillatory motion of the circular domain, induced by the feedback strategies, will couple to the characteristic modes and generate new flow patterns. For example, polymeric liquids show elastic turbulence due to an elastic instability [9]. One challenge will be to stabilize a regular periodic flow pattern in this turbulent regime using time-delayed feedback as originally suggested by Pyragas [18].

We acknowledge support by the Deutsche Forschungsgemeinschaft in the framework of the collaborative research center SFB 910.

Open Access This is an open access article distributed under the terms of the Creative Commons Attribution License (http://creativecommons.org/licenses/by/4.0), which permits unrestricted use, distribution, and reproduction in any medium, provided the original work is properly cited.

\section{References}

1. H. Stone, A. Stroock, A. Ajdari, Annu. Rev. Fluid Mech. 36, 381 (2004).

2. T. Squires, S. Quake, Rev. Mod. Phys. 77, 977 (2005).

3. G.M. Whitesides, Nature 442, 368 (2006).

4. H. Amini, E. Sollier, M. Masaeli, Y. Xie, B. Ganapathysubramanian, H.A. Stone, D. Di Carlo, Nat. Commun. 4, 1826 (2013). 
5. D. Rothstein, E. Henry, J. Gollub, Nature 401, 770 (1999).

6. B. Thomases, M. Shelley, Phys. Rev. Lett. 103, 094501 (2009).

7. P.E. Arratia, C.C. Thomas, J. Diorio, J.P. Gollub, Phys. Rev. Lett. 96, 144502 (2006).

8. L. Pan, A. Morozov, C. Wagner, P.E. Arratia, Phys. Rev. Lett. 110, 174502 (2013).

9. A. Groisman, V. Steinberg, Nature 405, 53 (2000).

10. A. Groisman, V. Steinberg, Nature 410, 905 (2001).

11. G.I. Taylor, Philos. Trans. R. Soc. London, Ser. A 223, 289 (1923).

12. R. Bird, R. Armstrong, O. Hassager, Dynamics of Polymeric Liquids, Vol. 1, 2nd edition, Fluid Mechanics (A Wiley-Interscience Publication, John Wiley \& Sons, 1987).

13. A. Friedman, L.-S. Jiang, Commun. Part. Diff. Eq. 13, 515 (1988).

14. T. Seidman, Switching Systems and Periodicity (Springer, 1989).

15. P. Gurevich, W. Jäger, A. Skubachevskii, SIAM J. Math. Anal. 41, 733 (2009).

16. P. Gurevich, Discrete Cont. Dyn. Syst. 29, 1041 (2010).

17. P. Gurevich, S. Tikhomirov, J. Dyn. Differ. Eq. 23, 923 (2011).

18. K. Pyragas, Phys. Lett. A 170, 421 (1992).

19. P. Hövel, E. Schöll, Phys. Rev. E 72, 046203 (2005).

20. E. Schöll, G. Hiller, P. Hövel, M.A. Dahlem, Philos. Trans. R. Soc. London, Ser. A 367, 1079 (2009).
21. O. Lüthje, S. Wolff, G. Pfister, Phys. Rev. Lett. 86, 1745 (2001).

22. K. Lichtner, S.H.L. Klapp, EPL 92, 40007 (2010).

23. K. Lichtner, A. Pototsky, S.H.L. Klapp, Phys. Rev. E 86, 051405 (2012).

24. S.A.M. Loos, R. Gernert, S.H.L. Klapp, Phys. Rev. E 89, 052136 (2014).

25. D.A. Strehober, E. Schöll, S.H.L. Klapp, Phys. Rev. E 88, 062509 (2013).

26. O. Beck, A. Amann, E. Schöll, J. Socolar, W. Just, Phys. Rev. E 66, 016213 (2002).

27. S. Hata, H. Nakao, A.S. Mikhailov, EPL 98, 64004 (2012).

28. L.D. Landau, E.M. Lifshitz, Fluid Mechanics (Pergamon Press, 1987).

29. D.G. Duffy, Green's Functions with Applications (Taylor \& Francis, 2001)

30. K. Pyragas, Phys. Lett. A 206, 323 (1995).

31. R.G. Larson, E.S.G. Shaqfeh, S.J. Muller, J. Fluid Mech. 218, 573 (1990).

32. A.J. Bray, Philos. Trans. R. Soc. London, Ser. A 361, 781 (2003).

33. S. Berti, G. Boffetta, M. Cencini, A. Vulpiani, Phys. Rev. Lett. 95, 224501 (2005).

34. J. Liu, L. Dedè, J.A. Evans, M.J. Borden, T.J. Hughes, J. Comput. Phys. 242, 321 (2013). 